\title{
EVALUACIÓN AGRONÓMICA Y NUTRICIONAL DE OCHO LÍNEAS DE FRÍJOL CAUPÍ (Vigna unguiculata [L.] WALP.)
}

\author{
AGRONOMIC AND NUTRITIONAL EVALUATION \\ OF EIGHT LINES OF COWPEA BEAN (Vigna unguiculata [L.] WALP.)
}

\author{
${ }^{1}$ Carlos Enrique Cardona-Villadiego, ${ }^{2}$ Hermes Araméndiz-Tatis, \\ ${ }^{3}$ Carlos Enrique Cardona-Ayala, ${ }^{4}$ Miguel Mariano Espitia-Camacho \\ 1,2,3,4 Universidad de Córdoba, Montería, Colombia \\ ${ }^{1}$ carvil@fca.edu.co, ${ }^{2}$ haramendiz@correo.unicordoba.edu.co, \\ ${ }^{3}$ cecardona@correo.unicordoba.edu.co, ${ }^{4}$ mmespitia@correo.unicordoba.edu.co
}

\begin{abstract}
RESUMEN
Contextualización: el fríjol caupí es importante para la seguridad alimentaria de la región Caribe de Colombia, donde se cultivan genotipos criollos de bajo rendimiento por hectárea, susceptibles a factores bióticos y abióticos, y bajos contenidos de minerales.
\end{abstract}

Vacío de conocimiento: se desconoce el comportamiento agronómico y la acumulación de minerales en el grano, de nuevos genotipos obtenidos por selección individual.

Propósito del estudio: evaluar el comportamiento agronómico y la calidad nutricional de ocho líneas homocigotas avanzadas de fríjol caupí del programa de mejoramiento de la Universidad de Córdoba, Colombia.

Metodología: el experimento se estableció entre abril a julio de 2014, bajo un diseño en bloques completos aleatorizados con nueve tratamientos incluido el testigo Criollo-Córdoba, con cuatro repeticiones. Se evaluaron las características longitud de la vaina, longitud del grano, ancho del grano, peso de la vaina, número de semilla por vaina, peso de cien semillas, rendimiento de grano por hectárea y contenidos de hierro, zinc, fósforo y proteína.

Resultados y conclusiones: los genotipos presentaron diferencias significativas en número de semillas por vaina, longitud del grano, ancho de grano, rendimiento de grano por hectárea, contenido de hierro, fósforo y proteína en el grano. Las líneas L-042 y L-047 fueron las de mejor comportamiento agronómico y calidad nutricional. El análisis de componentes principales y el biplot permitieron identificar a los genotipos L-014 y L-047 asociados a longitud del grano, longitud de vaina y peso de 
vaina, mientras que L-006, L-042 y CR-CO, están asociados a ancho de grano, peso de cien semillas y rendimiento de grano por hectárea, lo cual es importante para los programas de mejoramiento genético.

Palabras clave: caracteres agronómicos; genotipos; proteína; rendimiento.

\section{ABSTRACT}

Contextualization: The cowpea bean is important for the food security of the Caribbean region of Colombia, where creole genotypes of low-yielding per hectare, susceptible to biotic and abiotic factors, and low mineral content are cultivated.

Knowledge gap: The agronomic performance and mineral accumulation in the grain of new genotypes obtained by individual selection are unknown.

Purpose: To evaluate the agronomic performance and nutritional quality of eight advanced homozygous lines of cowpea beans from the breeding program of the Universidad de Córdoba, Colombia.
Methodology: The experiment was established between April and July 2014, under a randomized complete block design with nine treatments, including the Criollo-Córdoba as control, with four replications. The characteristics pod length, grain length and width and, pod weight, number of seed per pod, weight of one hundred seed, grain yield per hectare, iron, zinc, phosphorus and protein content were evaluated.

Results and conclusions: The genotypes showed significant differences in pod weight, number of seed per pod, grain length, grain width, grain yield per hectares, iron, phosphorus and protein content in the grain. Lines L-042 and L-047 were the ones with the best agronomic performance and the highest nutritional quality. Principal component analysis and biplot allowed to identify the L-014 and L-047 genotypes associated with grain length, pod length and pod weight, while L-006, L-042 and CR-CO, are associated to grain width, weight of one hundred seed and grain yield per hectare, which is important for genetic improvement programs for this species.

Key words: agronomic characters; genotypes; protein; yield.

\section{RESUMEN GRÁFICO}

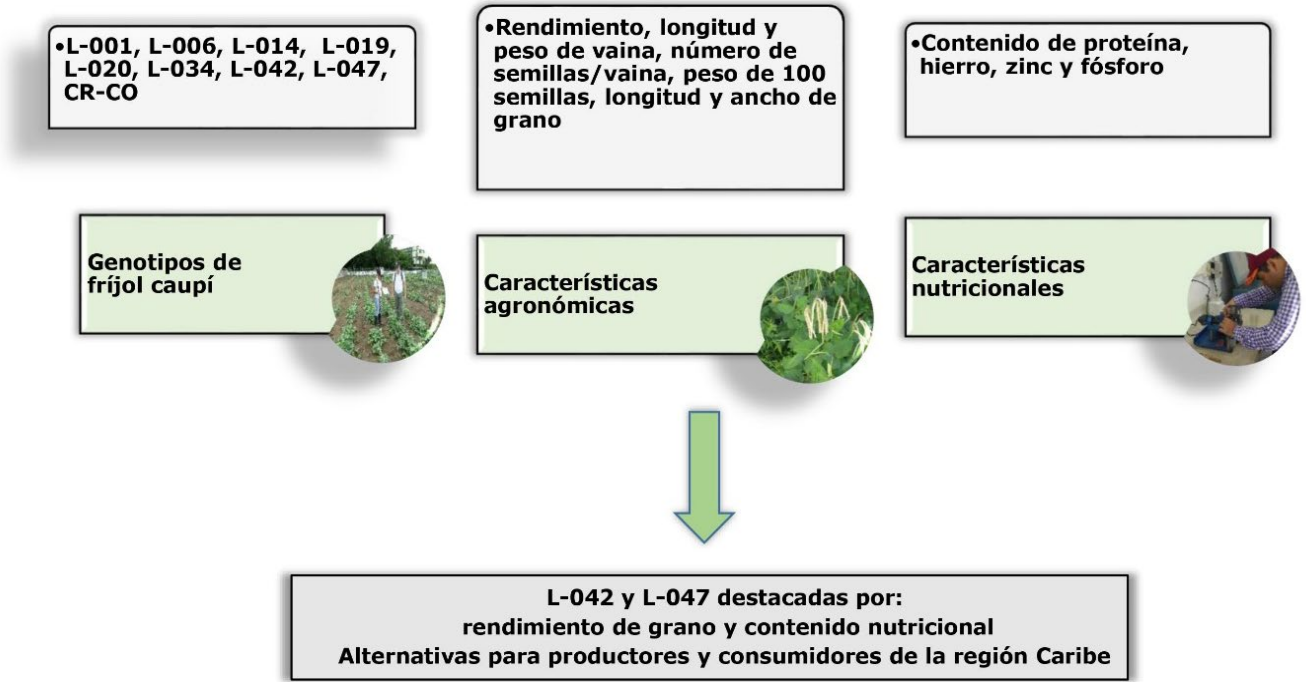

Fuente: autores 


\section{INTRODUCCIÓN}

El fríjol caupí (Vigna unguiculata [L.] Walp.) es una especie alimenticia importante en poblaciones de bajos ingresos de áreas tropicales y subtropicales del mundo (Guillén-Molina et al., 2016). Es utilizada por su valor proteico (Alidu, 2019) y contenido de hierro y zinc (Araméndiz-Tatis, Cardona-Ayala y Combatt-Caballero, 2016), es tolerante a sequía (Gerrano et al., 2018), y tiene capacidad de fijar nitrógeno y buen comportamiento en suelos de escasa fertilidad (Gerrano, Ransburg y Kutu, 2019). No obstante, a escala mundial, el rendimiento es de $400 \mathrm{~kg} \mathrm{ha}^{-1}$, por limitantes bióticos y abióticos (Jagadale, Bahure, Mirza, Mirche y Ghungarde, 2017).

El consumo de vegetales con bajo contenido de minerales genera hambre oculta, especialmente la deficiencia de hierro en poblaciones gestantes y en edad reproductiva, la deficiencia de zinc ocasiona atraso en el crecimiento, presión arterial alta y otras morbilidades (Zahir-Aziz et al., 2019). Esto demanda el mejoramiento de cultivares con ventajas nutricionales, agronómicas y económicas (Gerrano et al., 2019).

En Colombia, la producción de fríjol caupí está a cargo de pequeños productores, con restricciones tecnológicas, en áreas de 1.000 a 10.000 $\mathrm{m}^{2}$ (Cardona, Araméndiz y Jarma, 2013). En 2018, en el Departamento de Córdoba, se cosecharon 31 ha de fríjol caupí, con un rendimiento de 0,74 t ha-1 (Agronet, 2020). A pesar del rendimiento bajo del fríjol caupí, es importante como fuente de alimentación y de ingresos económicos en la región; y se consume de diversas formas (Araméndiz-Tatis et al., 2016).

En el mejoramiento genético de los cultivos, debe considerarse el rendimiento, la aceptación de los agricultores, el respeto por sus hábitos alimenticios y el contenido de minerales esenciales en el órgano de interés para un impacto positivo en la salud humana (Welch y Graham, 2005). Estudios sobre el fríjol común por Tofiño-Rivera-A, Pastrana-Vargas, MeloRíos, Beebe y Tofiño-Rivera-R (2016), con pequeños agricultores del Caribe seco de Colombia, reportaron ventajas de los genotipos biofortificados con relación al testigo.

El objetivo del estudio fue evaluar el comportamiento agronómico y los contenidos de hierro, zinc, fósforo y proteína de ocho líneas homocigotas de fríjol caupí más el cultivar Criollo Córdoba (CR-CO) como testigo, del programa de fitomejoramiento de la Universidad de Córdoba.

\section{MATERIALES Y MÉTODOS}

La investigación se desarrolló entre abril y julio de 2014 en la Universidad de Córdoba, ubicada en Montería-Colombia a los $8^{\circ} 44^{\prime} \mathrm{LN}$ y $75^{\circ} 53^{\prime} \mathrm{LO}$, a una altitud de $14 \mathrm{~m}$. Precipitación promedio de $1.300 \mathrm{~mm}$, con humedad relativa de $83 \%$, temperatura media de $28{ }^{\circ} \mathrm{C}$ y $6-7$ horas de luz por día.

Como material vegetal, se utilizaron las líneas: L-001, L-006, L-014, L-019, L-020, L-034, L-042, y CR-CO como testigo. Las líneas provienen de selección individual de la población heterogénea homocigota Criollo-Córdoba.

El experimento se realizó bajo el diseño de bloques completos al azar, con nueve genotipos y cuatro repeticiones. Cada parcela fue de $30,0 \mathrm{~m}^{2}$, formada por seis surcos de $5,0 \mathrm{~m}$ de largo, con un $1,0 \mathrm{~m}$ entre hileras y 0,5 m entre plantas. Se sembraron tres semillas por sitio, y ocho días después de la emergencia, se hizo raleo para dejar una plántula por sitio. El desarrollo de las plantas fue monitoreado diariamente, con buenas prácticas agrícolas 
(Organización de las Naciones Unidas para la Alimentación y la Agricultura [FAO], 2012).

\section{Variables agronómicas evaluadas}

Longitud de la vaina (LV): se midió la longitud, en centímetros, de cinco vainas en los cuatro surcos centrales por metro lineal tomadas al azar/parcela, y se obtuvo el promedio; Peso de la vaina (PV): se obtuvo el peso promedio, en gramos, de cinco vainas seleccionadas por/parcela, con una balanza analítica con precisión de 0,1 g; Número de semillas por vaina (NSV): se promedió el número de semillas de cinco vainas seleccionadas al azar por/parcela; Peso de cien semillas (PCS): se seleccionaron al azar 100 semillas sanas de cada parcela y se registró su peso (g); Longitud del grano (LG) y Ancho de grano (AG): se midió la longitud y el ancho, en centímetros, de 10 semillas tomadas al azar por/parcela; Rendimiento (Rha): se estimó el peso, en kilogramos, de la producción/parcela, expresado en t ha-1 y ajustado a $14 \%$ de humedad.

El contenido nutricional se avaluó en muestras de 2,0 $\mathrm{g}$ de semilla/parcela, en $\mathrm{mg} \mathrm{kg}^{-1}$ para hierro (Fe), zinc ( $\mathrm{Zn})$ y fósforo $(\mathrm{P})$, y se determinaron según los procedimientos AOAC 945.38, 969.32 y 931.01 , respectivamente (Association Official Analytical Chemists [AOAC], 2005).

La cuantificación se hizo por espectrofotometría de absorción atómica de llama, en un equipo Perkin Elmer 3110, para hierro y zinc; el fósforo, por absorción molecular en un Perkin Elmer XLS. El nitrógeno se determinó por digestión, destilación y titulación, según el método de Kjeldahl para granos AOAC 979.09 (Association Official Analytical Chemists $[A O A C], 2005)$. Se utilizó un equipo Buchi
K-355 y el contenido de proteína se cuantificó multiplicando el contenido de nitrógeno por el factor 6,25 .

\section{Análisis estadísticos}

Para los caracteres agronómicos, se realizaron análisis de varianza a dos vías y comparación de medias con la prueba de Tukey al 5\%. Para la calidad nutricional, se realizaron análisis de varianza a una vía y comparación de medias con la prueba DMS de Fisher al 5\%. Se verificó el cumplimiento de los supuestos de normalidad mediante la prueba de Shapiro-Wilk, y la homogeneidad de las varianzas de los errores con la prueba de Levene. Se realizó un análisis de componentes principales (ACP), desde la matriz de correlaciones, y selección de componentes principales con valores propios asociados mayores que 1,0. Para el análisis de los datos se utilizó el paquete estadístico SAS versión 9.2., y ACP biplot, para el que se usó el software libre MultBiplot (Villardón, 2018).

\section{RESULTADOS Y DISCUSIÓN \\ Características agronómicas}

El análisis de varianza detectó diferencias al $\mathrm{P}<0,05$ y $\mathrm{P}<0,001$ en cinco de las características agronómicas, excepto para LV y PCS (Tabla 1). Estos resultados evidencian variabilidad genética, la cual es importante para seleccionar genotipos con mejores características (Mofokeng, Mashilo, Rantso y Shimelis, 2020).

La ausencia de significancia en LV y PCS (Tabla 1), indica similitud de genes por la base genética estrecha al provenir de la misma población. Resultado contrario fue encontrado por Animasaun, Oyedeji, Azeez-Y, Mustapha y Azeez-MA (2015) y Lazaridi, Ntatsi, Savvas 
y Bebeli (2017) quienes estimaron diferencias significativas en poblaciones criollas de origen genético diferente. Los registros de LV son inferiores a los reportados por Silva et al. (2014), quienes sostienen que las vainas cortas tienen menos riesgo de contacto con el suelo, menor pudrición y facilitan la cosecha mecánica (Guerra, Carvalho, Medeiros, Souza y Brito, 2017).

La línea L-042 demostró mayor valor de PV, seguida por L-014, L-020 y L-047 (Tabla 1), las cuales fueron estadísticamente similares al testigo. Estos resultados son inferiores a los reportados por Teodoro et al. (2019), en cultivares de fríjol caupí originarios de Brasil. El PCS registró una media general superior a la reportada por António, Bofana, Gimo y Sueco (2019) y todos los genotipos presentaron valores dentro del rango encontrado por GuillénMolina et al. (2016).
EI NSV osciló entre 9,25 y 12,0 (Tabla 1), similar al reportado por Singh-V, Singh-AK, SinghMK, Raghuvanshi y Singh-U (2017) en India; sin embargo, Lazaridi et al. (2017), reportaron valores que oscilaron entre 4 y 16 , lo que se atribuye a la diferencia en la cantidad de óvulos fecundados.

Los valores de LG oscilaron entre 0,76 y 0,96 $\mathrm{cm}$, con el genotipo L-020 sobresaliendo con el mayor valor, mientras que L-001 y L-006, registraron el menor. Este resultado coincide con los trabajos de Morales-Morales et al. (2019) y evidencia ventaja competitiva para L-20, por el tamaño y por el color crema de la testa apetecido en el mercado nacional e internacional. El ancho del grano (AG) fue similar en los genotipos L-042, L-020, L-047 y CR-CO (Tabla 1), lo que corrobora la ventaja de L-020, para su comercialización por su mayor tamaño.

Tabla 1. Medias de características agronómicas y cuadrados medios de nueve genotipos de fríjol caupí.

\begin{tabular}{|c|c|c|c|c|c|c|c|c|c|c|c|c|}
\hline \multicolumn{13}{|c|}{ GENOTIPOS } \\
\hline $\mathrm{CA}$ & L-001 & L-006 & L-014 & L-019 & L-020 & L-034 & L-042 & L-047 & $\mathrm{CR}-\mathrm{CO}$ & MG & CMT & CV \\
\hline LV & $14,87 \mathrm{a}$ & $14,02 \mathrm{a}$ & $15,47 \mathrm{a}$ & $14,40 a$ & 15,97 a & $14,12 \mathrm{a}$ & $15,75 \mathrm{a}$ & $14,95 \mathrm{a}$ & $14,57 \mathrm{a}$ & 14,9 & $1,97 \mathrm{~ns}$ & 8,3 \\
\hline PV & $1,90 a b$ & $1,67 b$ & $2,17 a b$ & $1,77 a b$ & $2,07 a b$ & $1,95 a b$ & $2,27 \mathrm{a}$ & $2,07 a b$ & $1,97 \mathrm{ab}$ & 2,0 & $0,14 *$ & 10,9 \\
\hline NSV & $11,50 \mathrm{a}$ & $10,00 \mathrm{a}$ & $11,75 \mathrm{a}$ & $10,75 \mathrm{a}$ & 9,25 a & $12,00 \mathrm{a}$ & $9,75 \mathrm{a}$ & $10,00 \mathrm{a}$ & $11,00 \mathrm{a}$ & 10,7 & $3,80 *$ & 11,0 \\
\hline PCS & $14,87 \mathrm{a}$ & $17,12 \mathrm{a}$ & $15,30 \mathrm{a}$ & $15,72 \mathrm{a}$ & $14,70 \mathrm{a}$ & $15,22 \mathrm{a}$ & $16,75 \mathrm{a}$ & $15,02 \mathrm{a}$ & $16,97 \mathrm{a}$ & 15,7 & $3,60 \mathrm{~ns}$ & 13,3 \\
\hline LG & $0,76 \mathrm{~b}$ & $0,76 \mathrm{~b}$ & $0,79 b$ & $0,78 \mathrm{~b}$ & $0,96 \mathrm{a}$ & $0,76 \mathrm{~b}$ & $0,80 a b$ & $0,86 a b$ & $0,84 a b$ & 0,82 & $0,02 * *$ & 8,2 \\
\hline$A G$ & $0,59 \mathrm{~d}$ & $0,61 \mathrm{~cd}$ & $0,62 \mathrm{~cd}$ & $0,59 \mathrm{~d}$ & $0,62 \mathrm{~cd}$ & $0,60 \mathrm{~d}$ & 0,67 a & $0,66 a b$ & $0,65 a b c$ & 0,63 & $0004 * *$ & 3,4 \\
\hline Rha & $0,91 a b$ & $0,87 a b$ & $0,71 a b$ & $0,89 a b$ & $0,39 \mathrm{~b}$ & $0,87 a b$ & $1,11 \mathrm{a}$ & $1,00 a b$ & $0,91 \mathrm{ab}$ & 0,86 & $0,16 *$ & 30,3 \\
\hline
\end{tabular}

CA: características agronómicas; MG: media general; CMT: cuadrados medios de genotipos; CV: coeficiente de variación (\%); * y **: diferencias estadísticas significativas a $\mathrm{P}<0,05$ y $\mathrm{P}<0,001$, respectivamente; ns: no significativo. LV: Iongitud de vaina (cm); PV: peso de vaina (g); NSV: número de semillas/vaina; PCS: peso de cien semillas

(g); LG: longitud del grano $(\mathrm{cm})$; AG: ancho del grano $(\mathrm{cm})$; Rha: rendimiento de grano ( $\mathrm{t}$ ha ${ }^{-1}$ ). Medias con letras iguales en la misma hilera no difieren significativamente (Prueba de Tukey al $5 \%$ ).

Fuente: autores 
Tabla 2. Contenidos de hierro, zinc y fósforo $\left(\mathrm{mg} \mathrm{kg}^{-1}\right)$, proteína (\%), y cuadrados medios de nueve genotipos de fríjol caupí.

\begin{tabular}{|c|c|c|c|c|c|c|c|c|c|c|c|c|}
\hline \multicolumn{13}{|c|}{ Medias de genotipos y cuadrados medios } \\
\hline EM & L-001 & L-006 & L-014 & L-019 & L-020 & L-034 & L-042 & L-047 & $\mathrm{CR}-\mathrm{CO}$ & MG & CMT & $\mathrm{CV}$ \\
\hline $\mathrm{Fe}$ & $45,8 \mathrm{~cd}$ & $45,8 \mathrm{~cd}$ & $39,0 d$ & $47,6 b c$ & $49,7 a b c$ & $48,4 a b c$ & $54,1 a b$ & $54,6 a$ & $53,90 a b$ & 48,8 & $102,5 * *$ & 9,5 \\
\hline $\mathrm{Zn}$ & $53,2 a$ & $54,5 a$ & $52,6 a$ & $55,0 a$ & $53,3 a$ & $55,2 a$ & $54,8 a$ & $53,4 a$ & $55,12 a$ & 54,1 & $4,0 \mathrm{~ns}$ & 7,6 \\
\hline$P$ & $3,3 \mathrm{de}$ & $3,3 e$ & $3,5 \mathrm{~cd}$ & $3,2 e$ & $3,8 b$ & $3,7 \mathrm{bc}$ & $4,3 a$ & $4,2 a$ & $3,60 \mathrm{~cd}$ & 3,7 & $0,61 * *$ & 4,5 \\
\hline PR & $26,7 b$ & $26,1 \mathrm{bc}$ & $24,3 d$ & $25,9 c$ & $25,9 c$ & $26,5 b c$ & $28,8 a$ & $28,4 a$ & $26,80 b$ & 26,6 & $7,4^{* *}$ & 2,2 \\
\hline
\end{tabular}

EM: elemento mineral; Fe: hierro; Zn: zinc; P: fósforo; PR: proteína (\%); MG: media general; CMT: cuadrados medios de genotipos; CV: coeficiente de variación (\%); **: diferencias estadísticas significativas a $\mathrm{P}<0,001$; ns: no significativo. Medias con letras iguales en la misma hilera no difieren significativamente (prueba DMS al 5\%).

Fuente: autores

El análisis de varianza para el rendimiento (Rha) estimó diferencias significativas entre genotipos (Tabla 1), derivadas de las divergencias genéticas. Se identificaron genotipos que rindieron por encima de los $1000 \mathrm{~kg} \mathrm{ha}^{-1}$, lo cual es una ventaja comparativa y representan un uso potencial para los productores. La prueba de medias determinó que L-042 triplicó al genotipo L-020, pero estadísticamente fue similar al resto de cultivares. Estos resultados son ligeramente superiores a los reportados por Gerrano et al. (2019) en Sudáfrica, y Agyeman, Berchie, Osei-Bonsu, Tetteh y Fordjour (2015) en Ghana.

\section{Contenido nutricional del grano}

El análisis para el contenido de hierro presentó significancia $(P<0,001)$ entre los genotipos (Tabla 2), siendo la línea L-047 la de mayor concentración, con 54,6 mg kg-1; mientras que la línea L-014, la menor con 39,0 mg kg-1. Esta variación podría obedecer a la capacidad de profundización de raíces y toma de nutrientes para su traslado al órgano de interés (Singh et al., 2017). Los contenidos de hierro son superiores a los reportados por Guillén-Molina et al. (2016), pero inferiores al fríjol común (Zemolin, Ribeiro, Casagrande, Silva y Arns, 2016). El contenido de hierro de L-047, L-042 y
Criollo-Córdoba fue superior al de L-014, pero similar al de L-020, L-034, L-019, L-006 y L-001 (Tabla 2). Estos contenidos pueden satisfacer la demanda diaria de niños, mujeres embarazadas y, en general, de adultos (USDA, 2016).

El análisis de varianza para zinc no mostró significancia entre genotipos (Tabla 2), posiblemente por su base genética común. Los contenidos superiores a $52,6 \mathrm{mg} \mathrm{kg}^{-1}$, superan las estimaciones Gerrano et al. (2018), lo cual es importante en la salud pública. Esto sugiere que el consumo de estos genotipos es ventajoso para reducir los problemas de carencia de zinc de poblaciones vulnerables, por la denominada malnutrición proteinoenergética (Hurtado-Quintero et al., 2016), ya que satisface las demandas biológicas.

Para el fósforo, el análisis de varianza mostró diferencias $(P<0,001)$ entre genotipos (Tabla 3). El contenido de este elemento varió de 3,2 a $4,3 \mathrm{mg} \mathrm{kg}^{-1}$. El genotipo L-042 presentó el mayor contenido; mientras que L-019, el menor. La acumulación de fósforo en el grano obedece a la disponibilidad del elemento en el suelo, y la capacidad de absorción y transporte. Estos registros son inferiores a los reportados por Gerrano et al. (2018) respecto al 
fríjol caupí, y su ingesta permite superar las deficiencias de fósforo en personas muy desnutridas, alcohólicas o con hipofosfatemia $(<3$ $\mathrm{mg} / \mathrm{dl}$ ) (USDA, 2016).

El análisis de varianza para proteína reveló diferencias $(P<0,001)$ entre los tratamientos (Tabla 2). El contenido de proteína fue mayor en las líneas L-042 y L-047, con porcentaje superior al $28 \%$, lo cual es muy relevante para las poblaciones vulnerables, por el aporte de aminoácidos esenciales en la alimentación.

\section{Análisis de componentes principales (ACP)}

El análisis de componentes principales determinó que, con los tres primeros componentes, se explica un $89,6 \%$ de la variabilidad total observada, y cada una posee un valor propio superior a 1,0 (Tabla 3), criterio considerado por Gerrano et al. (2018) y Mofokeng et al. (2020).

El CP1 retiene el $44,76 \%$ de la variabilidad total y está representado por las variables asociadas al PV, LV y LG (Tabla 3). Esto indica que la formación de vainas de mayor peso y longitud, con granos largos, son las variables que más contribuyen a este componente. El $\mathrm{CP} 2$ retiene el $27,8 \%$ de la variabilidad total, y las variables que más contribuyeron fueron el PCS, AG y Rha.

Por último, el CP3 retiene el $17,0 \%$ de la variabilidad total y está más influenciado por el NSV. En general, los dos primeros componentes explican el $72,6 \%$ de la variabilidad estimada y contribuyen eficazmente a la interpretación del ACP-biplot (Figura 1), que representa las siete características agronómicas asociadas a los genotipos (Mofokeng et al., 2020).

En el ACP-biplot (Figura 1) L-014 y L-047 están asociados a LG, LV y PV, y se oponen a los genotipos L-001, L-019 y L-034 que están asociados a NSV. Por otro lado, los genotipos L-006, L-042 y CR-CO, constituyen otro grupo asociado a $A G, P C S$ y Rha.

Tabla 3. Valores y vectores propios de los tres primeros componentes principales para siete características agronómicas de nueve genotipos de fríjol caupí.

\begin{tabular}{|c|c|c|c|c|c|c|c|}
\hline \multirow[b]{2}{*}{ No. } & \multicolumn{3}{|c|}{ Valores propios (Varianzas) } & \multirow[b]{2}{*}{ Características } & \multicolumn{3}{|c|}{ Vectores propios } \\
\hline & $\mathbf{v}$ & $\% \mathbf{v}$ & \%VA & & CP1 & CP2 & CP3 \\
\hline 1 & 3,13 & 44,7 & 44,7 & LV & 0,513 & $-0,04$ & 0,220 \\
\hline 2 & 1,95 & 27,8 & 72,6 & PV & 0,426 & 0,172 & 0,544 \\
\hline 3 & 1,19 & 17,0 & 89,6 & NSV & $-0,353$ & $-0,170$ & 0,608 \\
\hline 4 & 0,38 & 5,4 & 95,0 & PCS & $-0,152$ & 0,550 & $-0,363$ \\
\hline 5 & 0,29 & 4,2 & 99,2 & LG & 0,486 & $-0,168$ & $-0,302$ \\
\hline 6 & 0,04 & 0,6 & 99,8 & $A G$ & 0,345 & 0,535 & 0,053 \\
\hline 7 & 0,02 & 0,2 & 100,0 & Rha & $-0,231$ & 0,569 & 0,246 \\
\hline
\end{tabular}

V: varianza de cada eje; \%V: porcentaje de la varianza total; \%VA: porcentaje acumulado de la varianza total; $\mathrm{CP} 1, \mathrm{CP} 2$ y CP3: vectores propios con ponderaciones para cada característica. 


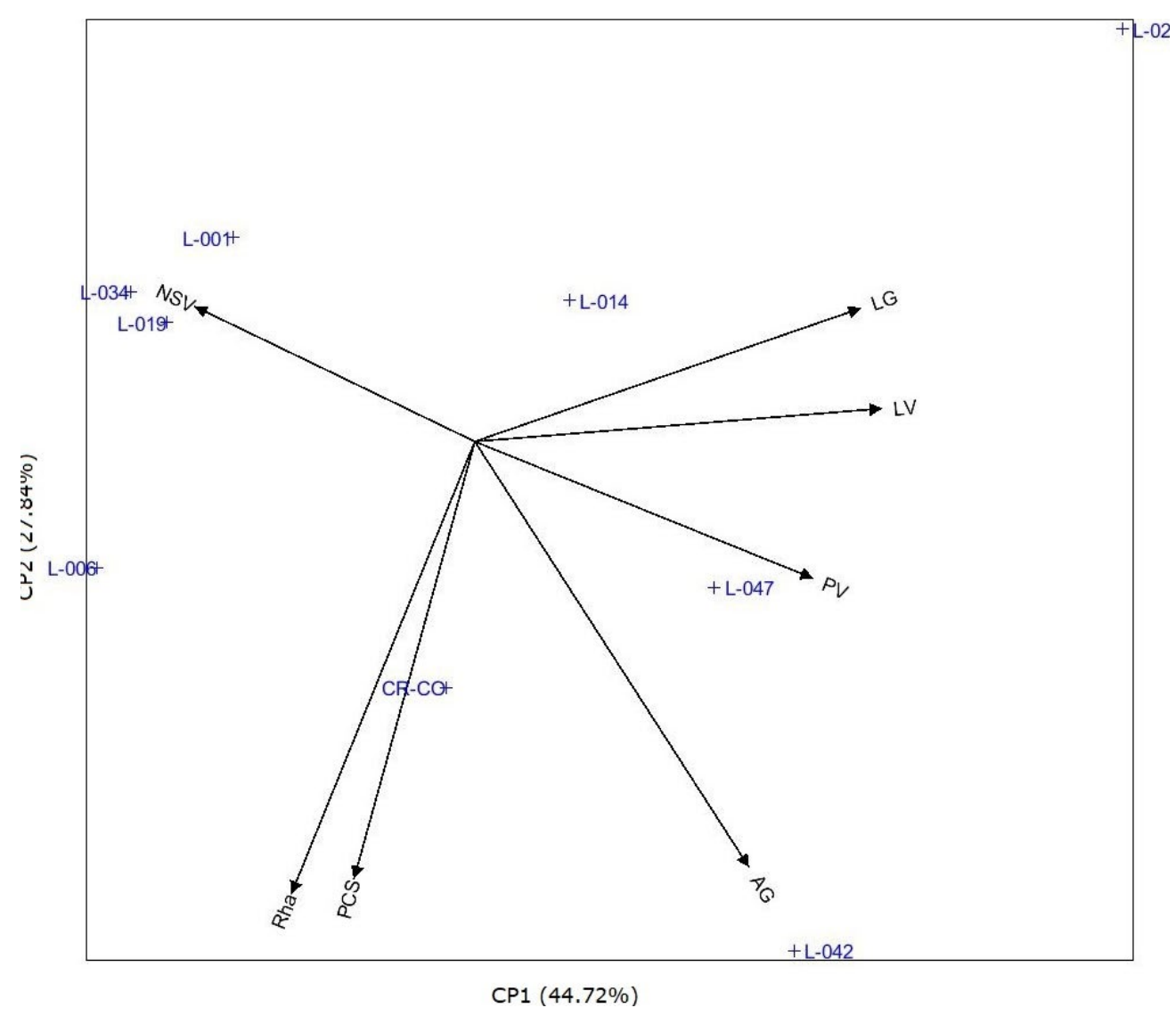

Figura 1. ACP-Biplot para siete características agronómicas de nueve genotipos de fríjol caupí.

Fuente: autores

\section{CONCLUSIONES}

Las líneas L-042 y L-047 presentaron la mejor combinación de rendimiento de grano y contenidos de hierro, fósforo y proteína, y representan una alternativa para productores y consumidores de la región Caribe.

El ACP y el biplot permitieron identificar genotipos asociadas con características agronómicas específicas, lo cual es importante para el mejoramiento genético de la especie.

\section{CONTRIBUCIÓN DE LA AUTORÍA}

Primer autor: metodología, investigación, base de datos, escritura borrador original.

Segundo autor: administrador del proyecto, metodología, investigación, y edición.

Tercer autor: investigación, análisis de datos, escritura-revisión y edición.

Cuarto autor: logística, investigación, revisión y edición.

\section{AGRADECIMIENTOS}

Los autores expresan sus agradecimientos a la UNIVERSIDAD DE CORDOBA por la financiación del proyecto "Obtención y entrega de cultivares de fríjol caupí (Vigna unguiculata (L.) Walp) biofortificados con adaptación a los efectos del cambio climático en el caribe colombiano" código FCA 01-11, numeral 1.2.08.110.18 y al personal de apoyo del Laboratorio de Fitomejoramiento de la misma institución. 


\section{LITERATURA CITADA}

Agronet. (2020). Reporte: Área, Producción y Rendimiento Nacional por Cultivo. Agronet, MinAgricultura. Recuperado de https://www.agronet. gov.co/estadistica/Paginas/home.aspx?cod=1.

Agyeman K., Berchie, J., Osei-Bonsu, I., Tetteh, E., y Fordjour, J. (2015). Seed yield and agronomic performance of seven improved cowpea (Vigna unguiculata L.) varieties in Ghana. African Journal of Agricultural Research, 10(4), 215-221.

Alidu, S.T. (2019). Evaluation of Planting Dates on Growth and Yield of Three Cowpea [Vigna unguiculata (L) Walp.] Genotypes in Northern Ghana. Advances in Research, 18(4), 1-14.

Animasaun, D., Oyedeji, S., Azeez, Y., Mustapha, O.T., y Azeez, M.A. (2015). Genetic Variability Study Among Ten Cultivars of Cowpea (Vigna unguiculata L. Walp) Using Morpho-agronomic Traits and Nutritional Composition. The Journal of Agricultural Sciences, 10(2), 119-130. http:// doi.org/10.4038/jas.v10i2.8057

António, G.H., Bofana, J., Gimo, F., y Sueco, A.C. (2019). Evaluation of Grain Yield of Two Varieties of Cowpea (Vigna unguiculata) Subjected to Four Agricultural Practices in Gùrué District, Mozambique. Journal of Experimental Agriculture International, 36(5), 1-7. doi.org/10.9734/jeai/2019/ v36i530247

Araméndiz-Tatis, H., Cardona-Ayala, C., y CombattCaballero, E. 2016. Contenido Nutricional de Líneas de Fríjol Caupí (Vigna unguiculata L. Walp.) Seleccionadas de una Población Criolla. Información Tecnológica, 27(2),53-60. doi: http://dx.doi. org/10.4067/S0718-07642016000200007

Association Official Analytical Chemists. (2005). Official Methods of Analysis of the Association of Official Analytical Chemists. Vol. 1. 18 ${ }^{\text {th }}$. Edition. Association of Official Analytical Chemists, Washington DC, USA.

Cardona, C.E., Araméndiz-Tatis, H., y Jarma, A. (2013). Variabilidad genética en líneas de fríjol caupí (Vigna unguiculata L. WALP). Agronomía, 21(2), 7-18.

Gerrano, A.S., Ransburg, W.S., Venter, S.L., Shargie, N.G., Amelework, B.A., Shimelis, H.A., y Labuschagne, M.T. (2018). Selection of cowpea genotypes based on grain mineral and total protein content. Acta Agriculturae Scandinavica, Section B - Soil \& Plant Science, 69(2),
155-166. doi: https://doi.org/10.1080/0906471 0.2018 .1520290

Gerrano, A.S., Rensburg, W.S., y Kutu, F.R. (2019). Agronomic evaluation and identification of potential cowpea (Vigna unguiculata L. Walp) genotypes in South Africa. Acta Agriculturae Scandinavica, Section B - Soil \& Plant Science, 69 (4), 295-303. doi: https://doi.org/10.1080/09064710 .2018 .1562564

Guerra, J.V., Carvalho, A.J., Medeiros, J.C., Souza, A.A., y Brito, O.G. (2017). Agronomic performance of erect and semi-erect cowpea genotypes in the north of minas gerais, Brazil. Revista Caatinga, 30 (3), 679 - 686. doi.org/10.1590/198321252017v30n316rc

Guillén-Molina, M., Márquez-Queiroz, C., Cruz-Lázaro, E., Velásquez-Martínez, J.R., Soto-Parra, J.M., García-Carrillo, M., y Orozco-Vidal, J.A. (2016). Biofortificación de frijol caupí (Vigna unguiculata L. Walp) con hierro y zinc. Revista Mexicana de Ciencias Agrícolas, 17, 3427-3438. doi: https:// doi.org/10.29312/remexca.v0i20.986

Hurtado-Quintero, C., Mejía, C., Mejía, F., Arango, C., Chavarriaga, L.M., y Grisales-Romero H. (2016). Malnutrición por exceso y déficit en niños, niñas y adolescentes, Antioquia, 2015. Revista Facultad Nacional de Salud Pública, 35(1), 58-70. doi.org/10.17533/udea.rfnsp.v35n1a07

Jagadale, A.R., Bahure, G.K., Mirza, I.A.B., Mirche, S.H., y Ghungarde, S.R. (2017). Effect of Plant Geometry and Fertilizer Levels on Yield and Economic of Cowpea (Vigna unguiculata L. Walp). International Journal of Current Microbiology and Applied Sciences, 6(5), 1518-1522. doi: https:// doi.org/10.20546/ijcmas.2017.605.165

Lazaridi, E., Ntatsi, G., Savvas, D., y Bebeli, P.J. (2017). Diversity in cowpea (Vigna unguiculata (L.) Walp.) local populations from Greece. Genetic Resources and Crop Evolution, 64: 15291551. doi.org/10.1007/s10722-016-0452-6

Mofokeng, M.A., Mashilo, J., Rantso, J.M., y Shimelis, H. (2020). Genetic variation and genetic advance in cowpea based on yield and yield-related traits. Acta Agriculturae Scandinavica, Section B - Soil \& Plant Science, 69(4), 295-303. doi.org/1 $\underline{0.1080 / 09064710.2020 .1749295}$

Morales-Morales, A., Andueza, R., Márquez, C., Benavides, A., Tun, J., González, A., y Alvarado, C. (2019). Caracterización morfológica de 
semillas de frijol caupí (Vigna unguiculata L. Walp) de la Península de Yucatán. Ecosistemas y Recursos Agropecuarios, 6(18), 463-475. doi. org/10.19136/era.a6n18.2171

Organización de las Naciones Unidas para la Agricultura y la Alimentación (2012). Manual de Buenas Prácticas Agrícolas para el Productor Hortofrutícola. Recuperado de https://www.google. $\mathrm{com} /$ search?client $=$ firefox-b-d\&ei=UNWQX5P5 BZGB5wKygr3QBA\&q=Manual+de+buenas+pr $\%$ C3\%A1cticas+agr\%C3\%ADcolas+FAO +2012 \&oq =Manual+de+buenas + pr\%C3\%A1cticas+agr\%C3\%ADcolas+FAO+2012\&gs_Icp $=$ CgZwc3 ktYWIQAzoECAAQRzoGCAAQFhAeULiyAliMxgJgy skCaABwAngAgAHMAYgBnQuSAQUwLjcuMZgBA KABAaoBB2d3cy13aXrIAQjAAQE\&sclient $=p s y-a$ b\&ved=0ahUKEwiT4ZXX-8bsAhWRwFkKHTJBD0 oQ4dUDCAw\&uact $=5$

Silva, A.C., Morais, O.M., Santos, J., Aredes, L., Silva, C., y Rocha, M. (2014). Estimates of genetic parameters in Vigna unguiculata. Revista de Ciências Agrárias, 37 (4), 399-407.

Singh, V., Singh, A.K., Singh, M.K., Raghuvanshi, T., y Singh, U. (2017). Morphological and Yield Traits of Cowpea (Vigna unguiculata L. Walp.) under Integrated Nutrient Management. International Journal of Current Microbiology and Applied Sciences, 6 (10), 3402-3407. doi.org/10.20546/ ijcmas.2017.610.399

Teodoro, L.P., Evangelista, J.S., Damacena, M.B., Elizeu, A., Coelho, I.F., Rodrigues, E.V., y Teodoro, P.E. (2019). Estimates of genetic divergence in cowpea by multivariate analysis in different environments. Bioscience Journal, 35 (6), 16811687. doi.org/10.14393/BJ-v35n6a2019-42406
Tofiño-Rivera, A., Pastrana-Vargas, I., Melo-Ríos, A., Beebe, S., y Tofiño-Rivera, R. (2016). Rendimiento, estabilidad fenotípica y contenido de micronutrientes de fríjol biofortificado en el Caribe seco colombiano. Ciencia \& Tecnología Agropecuaria, 17(3), 309-329. doi.org/10.21930/rcta. vol17_num3_art:511

USDA U.S. Department of Agriculture. (2016). Food and Nutrition Information Center. Recuperado de https://www.nal.usda.gov/fnic/Dietary-Guidelines

Villardón, J.L. (2018). MultBiplot Multivariate Analysis using Biplots. Departamento de Estadística. Universidad de Salamanca. http://biplot.usal.es/ multbiplot/download/

Welch, R.M. yb Graham, R.D. (2005). Agriculture: the real nexus for enhancing bioavailable micronutrients in food crops. Journal of Trace Elements in Medicine and Biology, 18 (4), 299-307. doi.org/10.1016/j.jtemb.2005.03.001

Zahir-Aziz, M., Yaseen, M., Abbas, T., Naveed, M., Mustafa, A., Hamid, Y., Aeed, Q., y Ming-Gang, X. (2019). Foliar application of micronutrients enhances crop stand, yield and the biofortification essential for human health of different wheat cultivars. Journal of Integrative Agriculture, 18(6), 1369-1378. doi: https://doi.org/10.1016/ $\underline{\text { S2095-3119(18)62095-7 }}$

Zemolin, A.E., Ribeiro, N.D., Casagrande, C.R., Silva, M., y Arns, F.D. (2016). Genetic parameters of iron and zinc concentrations in Andean common bean seeds. Acta Scientiarum. Agronomy, 38 (4), 439-446. doi.org/10.4025/actasciagron. v38i4.30652
Conflicto de Intereses Los autores declaran no tener ningún conflicto de intereses 